\title{
Are We Reaching the Limits of Homo sapiens?
}

\begin{abstract}
Adrien Marck ${ }^{1,2}$, Juliana Antero ${ }^{1}$, Geoffroy Berthelot ${ }^{1,3,4}$, Guillaume Saulière ${ }^{1}$, Jean-Marc Jancovici ${ }^{5}$, Valérie Masson-Delmotte ${ }^{6}$, Gilles Boeuf ${ }^{7}$, Michael Spedding ${ }^{8}$, Éric Le Bourg ${ }^{9}$ and Jean-François Toussaint ${ }^{1,3,10 *}$

'Institut de Recherche bio-Médicale et d'Epidémiologie du Sport (IRMES) EA 7329, Institut National du Sport, de l'Expertise et de la Performance, Université Paris Descartes, Université Sorbonne Paris Cité, Paris, France, ${ }^{2}$ Laboratoire Matière et Systèmes Complexes, UMR 7057 Université Paris Diderot, Centre National de la Recherche Scientifique, Université Sorbonne Paris Cité, Paris, France, ${ }^{3}$ Group Adaptation and Prospective, High Council of Public Health, Paris, France, ${ }^{4}$ Research Laboratory for Interdisciplinary Studies, Paris, France, ${ }^{5}$ Carbone 4, Paris, France, ${ }^{6}$ Laboratoire des Sciences du Climat et l'Environnement, Institut Pierre Simon Laplace, CEA-Centre National de la Recherche Scientifique, Université de Versailles Saint-Quentin en Yvelines, Gif-sur-Yvette, France, ${ }^{7}$ Muséum National d'Histoire Naturelle, Université Pierre et Marie Curie, Sorbonne Universités, Paris, France, ${ }^{8}$ IUPHAR and Spedding Research Solutions SAS, Le Vésinet, France, ${ }^{9}$ Centre de Recherches sur la Cognition Animale, Centre de Biologie Intégrative, Université de Toulouse, Centre National de la Recherche Scientifique, Université Toulouse III Paul Sabatier, Toulouse, France, ${ }^{10}$ Centre d'Investigations en Médecine du Sport (CIMS), Hôtel-Dieu de Paris, Assistance Publique - Hôpitaux de Paris, Paris, France
\end{abstract}

Echoing scientific and industrial progress, the Twentieth century was an unprecedented period of improvement for human capabilities and performances, with a significant increase in lifespan, adult height, and maximal physiological performance. Analyses of historical data show a major slow down occurring in the most recent years. This triggered large and passionate debates in the academic scene within multiple disciplines; as such an observation could be interpreted as our upper biological limits. Such a new phase of human history may be related to structural and functional limits determined by long term evolutionary constraints, and the interaction between complex systems and their environment. In this interdisciplinary approach, we call into question the validity of subsequent forecasts and projections through innovative and related biomarkers such as sport, lifespan, and height indicators. We set a theoretical framework based on biological and environmental relevance rather than using a typical single-variable forecasting approach. As demonstrated within the article, these new views will have major social, economical, and political implications.

Keywords: anthropocene, human upper limits, performance, longevity, life span, biometry, public health, environment

\section{INTRODUCTION}

How long can we live (Olshansky et al., 1990, 2001; Aarssen and de Haan, 1994; Wilmoth, 1998; Thatcher, 1999; Oeppen and Vaupel, 2002; Watts et al., 2006; Carnes and Olshansky, 2007; Hayflick, 2007; Christensen et al., 2009; Olshansky and Carnes, 2009, 2013; Weon and Je, 2009; Vaupel, 2010; Couzin-Frankel, 2011; Li et al., 2011; Beltrán-Sánchez et al., 2012; Bravo and Real, 2012; Le Bourg, 2012; da Silva Antero-Jacquemin et al., 2014; Finch et al., 2014; Dong et al., 2016; Hanayama and Sibuya, 2016; Brown et al., 2017; de Beer et al., 2017; Gavrilov et al., 2017; Gbari et al., 2017; Hughes and Hekimi, 2017; Kirkwood, 2017; Le Bourg and Vijg, 2017; Lenart and Vaupel, 2017; Rootzén and Zholud, 2017; Rozing et al., 2017; Vijg and Le Bourg, 2017)? How fast can we run or swim 
(Kennelly, 1906; Hill, 1925; Deakin, 1967; Chatterjee and Chatterjee, 1982; Whipp and Ward, 1992; Blest, 1996; Reinboud, 2004; Tatem et al., 2004; Nevill and Whyte, 2005; Kuper and Sterken, 2007; Nevill et al., 2007; Berthelot et al., 2008, 2010b, 2015; Denny, 2008; Desgorces et al., 2008, 2012; Lippi et al., 2008; Weyand et al., 2010; Liu et al., 2012; Haake et al., 2014; Haugen et al., 2015; Kinugasa and Usami, 2016)? Demographers disagree about the lifespan trend and its potential limit, while sports scientists discuss the frontiers of maximal physical performance. Such questions stimulate large and passionate debates about the potential of Homo sapiens and its biological upper limits. Historical series, defined as the measurable data collected since the nineteenth century for lifespan, sport, or height provide crucial information to understand human physiology and the form and nature of our progression over the last 10 generations.

Recent studies about lifespan trends (da Silva AnteroJacquemin et al., 2014; Dong et al., 2016; Hughes and Hekimi, 2017) increased interest about the possible ceilings in longevity for humans. This long-lasting debate increased in strength at the beginning of the 1990s (Olshansky et al., 1990; Oeppen and Vaupel, 2002). Using biological and evolutionary arguments, the first leading opinion postulated an upper limit for life expectancy at birth and maximal longevity (Carnes and Olshansky, 2007; Hayflick, 2007; Olshansky and Carnes, 2009, 2013; Le Bourg, 2012). These limits may have already been approached: around 85-95 years for life-expectancy and 115-125 years for maximal longevity, as a result of nutritional, medical, societal, and technological progress (Carnes and Olshansky, 2007; Olshansky and Carnes, 2009; da Silva Antero-Jacquemin et al., 2014; Dong et al., 2016). A second school of thought considered that life expectancy may continue to progress indefinitely at a pace of 2 to 3 added years per decade (Oeppen and Vaupel, 2002; Christensen et al., 2009; Vaupel, 2010). They claim that most of the babies born during the 2000s, "if the present yearly growth in life expectancy continues through the twenty-first century," will celebrate their 100th birthday (Christensen et al., 2009) or, potentially reach physical immortality due to undefined scientific breakthroughs (de Grey, 2003; Kurzweil and Grossman, 2010).

Analyzing historical trends in sports series, a similar debate took place about physical performance upper limits (Kennelly, 1906; Hill, 1925; Whipp and Ward, 1992; Blest, 1996; Kuper and Sterken, 2003, 2007; Tatem et al., 2004; Nevill and Whyte, 2005; Nevill et al., 2007; Berthelot et al., 2008, 2010b, 2015; Denny, 2008). Since the former estimations of Kennelly (1906) or the pioneering work of Hill (1925), several studies have forecast future achievements (Blest, 1996; Nevill and Whyte, 2005; Nevill et al., 2007; Berthelot et al., 2008, 2010b, 2015). In one of the most extreme cases, Tatem and colleagues asserted in 2004 that performances progress linearly. They proposed that women would outrun men by 2156 in the $100 \mathrm{~m}$ track event (Tatem et al., 2004). A few studies also suggested continuous progression of records within upcoming decades or centuries (Whipp and Ward, 1992; Kinugasa and Usami, 2016; Rozing et al., 2017). Other authors considered that an upper limit gradually appears (Reinboud, 2004; Nevill and Whyte, 2005; Nevill et al., 2007; Berthelot et al., 2008, 2010a, 2015).
However, beyond mathematical projections and personal beliefs, these debates rarely integrate biological and environmental aspects of such trends (Carnes et al., 2014; Finch et al., 2014). Here, we will put such parameters into perspective as they shape common trends in lifespan, physical performance and height data. First, we will propose an overview of the body design and physical limits, as previously described in the longevity debate (Carnes and Olshansky, 2007; Olshansky and Carnes, 2009; Le Bourg, 2012), the structural and functional delimitations of the human organism integrating specific biological constraints resulting from evolutionary and environmental constraints. In light of these delimitations, we will then provide an analysis of the simultaneous progression of biometrical indicators under favorable environments. Finally, we will discuss the perspectives derived from the recently observed plateaus, which suggest upper biological limits for H. sapiens or an increase of its environmental constraints.

\section{LIMITED CAPABILITIES}

\section{Physically and Functionally Delimited Organism}

Most species share common features, including Mendelian inheritance (Dhar and Giuliani, 2010), individual growth and decline (e.g., the age-related changes in physical and cognitive performances) (Moore, 1975; Berthelot et al., 2011; Marck et al., 2016) and allometric scaling for energetic relationships (e.g., metabolic rate scales as the 3/4-power of mass) (Speakman, 2005; West and Brown, 2005). Following these emerging properties, individuals from our $H$. sapiens species are a delimited organism shaped by evolutionary constraints and modulated by their interactions with the environment.

The human body, as in any living species, is a finite organism, with structural (640 muscles and 206 bones) and functional boundaries at every level of organization. Each cell embeds around 20 to 25,000 protein-encoding genes over 2.85 billion nucleotides aligned among 46 chromosomes, shaping the human organism during development (Ezkurdia et al., 2014). From one cell at fecundation to $\sim 3 \times 10^{13}$ cells classified into more than 300 different types, the human organism constitutes a precisely delimited body (Bianconi et al., 2013).

Cells have a limited replicative potential depending on their type (Hayflick, 1965; Campisi, 1996). In addition, they accumulate damage with aging and replication, causing dysfunctions, while apoptotic and necrotic processes contribute to their gradual loss (Campisi, 1996). Such a process is common in muscle tissues that gradually lose fibers during aging, or among neurons or hematopoietic stem cells, further aggravating their functional decline (Mitchell et al., 2012; Holstege et al., 2014; Marck et al., 2016).

Each adult organ possesses capabilities and a particular size that is species-specific, with some variability (Poole and Erickson, 2011). For example, the normal human resting heart rate varies between 45 and 100 beats per minute (bpm), reaching a maximum of $220 \mathrm{bpm}$ during intensive effort (Tanaka et al., 2001; Mason et al., 2007; Nes et al., 2013). This maximal rate, which depends on age (Tanaka et al., 2001), is the upper 
functional performance of a normal human heart. Each organ has a similar age-dependent potential (Bassett and Howley, 2000); the maximal functional value usually reaches a peak during the third decade of life, and then gradually declines (Schoenberg et al., 1978; Wiswell et al., 2001; Mitchell et al., 2012; Kusy and Zielinski, 2014; Marck et al., 2016).

Such limitations of the human body and alterations with age are a subject of debate (de Grey, 2003; Carnes and Olshansky, 2007; Olshansky and Carnes, 2009; Kurzweil and Grossman, 2010). Prolongevists claim that aging could be delayed, slowed, reversed, or even eradicated during the next decades (de Grey, 2003; Carnes and Olshansky, 2007; Olshansky and Carnes, 2009; Kurzweil and Grossman, 2010; Vaupel, 2010). In sport, similar ideas suggest enhancement of the body's design, (e.g., by using gene therapy) (Berthelot et al., 2015). However, despite boisterous announcements, recent research has yet to deliver even one element showing how to enhance any human maximal performance.

\section{Long and Short Term Limitations through Evolutionary and Ecosystemic Constraints}

Recent human biometrical progression resulted from both long term human evolution and recent societal changes. It has been proposed that human evolution toward bipedalism and running may have paralleled climatic changes in Africa during the last three million years (Ruff, 1991; Bramble and Lieberman, 2004; Noakes and Spedding, 2012). It was associated with skeletal alterations and increased metabolic capacity as compared with non-human primates (Bramble and Lieberman, 2004; Lieberman and Bramble, 2007; Noakes and Spedding, 2012). At the same time, humans developed a large brain with spatial and social memory characteristics, which also resulted from a long adolescence (Neubauer and Hublin, 2012; Hublin et al., 2015). Changes in diet and more frequent meat-eating were critical (Domínguez-Rodrigo et al., 2010; Zaatari et al., 2016). A common driving force may have participated in the growth of both running and cognitive functions (Noakes and Spedding, 2012). Skeletal changes allowing high-speed throwing, important during hunting, also evolved $\sim 2$ million years ago (Roach et al., 2013). These changes form the background for many modern sports and physiological limits which have been derived from this evolutionary period.

Modern humans ventured out of Africa to occupy almost all terrestrial niches (Nielsen et al., 2017). Both biology and societies evolved and allowed humans to adapt to the most extreme surroundings. Recently, the industrial revolution deeply changed our environment progressively reducing its constraints on our daily habits, simultaneously triggering a global transition of living conditions and health in less than 10 generations (Wilmoth, 2000; Fogel, 2004; Omran, 2005; Steffen et al., 2007, 2011; McMichael, 2014). This was supported by a much higher primary energy consumption per capita (raised by one to two orders) and had large epidemiological (infections replaced by degenerative diseases as the main mortality cause), nutritional (less cereals, more meat, fat, and sugar in daily diets), agricultural (ten times higher yields), and demographical consequences (a mortality decrease followed by birth rate reduction a few decades later, allowing for the natural population increase) (Wilmoth, 2000;
Fogel, 2004; Omran, 2005; Steffen et al., 2007, 2011; McMichael, 2014).

During the nineteenth and twentieth centuries, human beings grew taller and lived longer (Wilmoth, 2000; Fogel, 2004; Olshansky and Carnes, 2009; Le Bourg, 2012; NCD-RisC, 2016). Life span and height echo development in many countries; their growth rates have been associated with energetic, nutritional, scientific, medical, and industrial progresses (Fogel, 2004; Omran, 2005; Le Bourg, 2012; Carnes et al., 2014; Finch et al., 2014; NCD-RisC, 2016; Stulp and Barrett, 2016). Likewise, metric or chronometric measurements of maximal physiological performances document such progress since the first modern Olympic Games in 1896. This allows for the development of accurate indicators and tools measuring human progression rates (Hill, 1925; da Silva Antero-Jacquemin et al., 2014; Berthelot et al., 2015).

These recent changes are highly dependent on phylogenetic and demographic constraints that provide a limited number of new phenotypic variants among recent generations. These generations inherit specific capabilities from the previous ones, limiting possible adaptations (Blomberg and Garland, 2002). On another timescale, physical and chemical constraints such as gravity or osmotic properties restrain the possible organism variations, most of them since the beginning of terrestrial life. As such, modern human potential, including an enlarged brain, height, lifespan, and physical performance has been dependent on very long-term evolutionary parameters (Kirkwood and Austad, 2000; Le Bourg, 2001; Bramble and Lieberman, 2004; Noakes and Spedding, 2012; Stulp and Barrett, 2016; Kirkwood, 2017; Le Bourg and Vijg, 2017; Vijg and Le Bourg, 2017). These constraints, applied to a singular genotype, shape a delimited organism in the course of its development, whose traits vary according to the possibilities of the species's genome. With such variability human height, for example, shows a one to five span (in centimeters) between the smallest and tallest adult individuals (from 55 to $272 \mathrm{~cm}$, both situations usually induce severe pathological conditions and major health risks) (Stulp and Barrett, 2016). However, these extreme phenotypes can be considered as the present lower and upper known limits of the potential height for $H$. sapiens.

In addition, population size is another factor that influences the progression of maximal biometrical values (Gillespie, 2001; Charlesworth, 2009; Foster et al., 2010; Lanfear et al., 2014). As the size of the population increases, the tails of the indicator distributions expand allowing extremely rare phenotypes, such as Jeanne Calment for longevity or Usain Bolt for maximal running speed (Williams and Folland, 2008).

Phenotypic plasticity depends on the environmental conditions; it is important in shaping both phenotypic changes in response to ecological alterations and ecological changes in response to phenotypic adaptation (DeWitt et al., 1998; Merilä and Hendry, 2014; Stulp and Barrett, 2016). Recent adult height evolution is an example of such plasticity, which primarily depended on nutrition availability, hygiene, and healthcare throughout infancy (NCD-RisC, 2016; Stulp and Barrett, 2016). In the last century, technological developments and their accelerated diffusion allowed for rapid phenotypic 
changes, with a major improvement in most physiological factors among humans and human-driven species (Fogel, 2004; Omran, 2005; Berthelot et al., 2015). This period covers a typical expansion of major physiological indicators that could be interpreted as a non-genetic "techno-physiological" evolution (Fogel, 2004) with a large phenotypic expansion (Berthelot et al., 2011) (epigenetic changes being more likely expected on such a brief time scale (Gapp et al., 2014; Bohacek and Mansuy, 2015; Fumagalli et al., 2015). This evolution was associated with a large increase of the energy available per capita (Fogel, 2004) and supported by a synergy between economic growth, medical advancements and technological diffusion inducing a positive feedback in the efficiency of energy production and use (Fogel, 2004; Steffen et al., 2007, 2011; Carnes et al., 2014; Finch et al., 2014; McMichael, 2014).

However, mankind is now the major actor implicated in its own environment alterations (Steffen et al., 2007, 2011; IPCC, 2014; McMichael, 2014). Sapiens alters his ecosystem, while the ecosystem also shapes him in return (Steffen et al., 2011). Our activities have been implicated as the dominant cause of most environment changes and the recent acceleration could have major impacts on human health, even if some progress has been recently made, such as the increased use of renewable energy (Steffen et al., 2007, 2011; IPCC, 2014; McMichael, 2014).

Physiological traits are directly and indirectly affected by environmental changes. For example, temperature plays a crucial role in mortality rates, in sport performances or in the interactions between species (Laaidi et al., 2006; El Helou et al., 2012; Grigaltchik et al., 2012; Haïda et al., 2013; Carnes et al., 2014; Finch et al., 2014; Berthelot et al., 2015). In running, best performances describe an inverted- $U$ shaped curve, with an optimal temperature at about $10^{\circ} \mathrm{C}$ for marathon and $23^{\circ} \mathrm{C}$ for sprint distances (100 m) (El Helou et al., 2012; Haïda et al., 2013; Berthelot et al., 2015). A similar relation links temperature and survival rates, with an optimum value between 20 and $26^{\circ} \mathrm{C}$ (Laaidi et al., 2006). Major temperature elevations during current climate changes may have unfavorable impacts on our capacity to reach our functional maxima and absolute physical limits.

Finally, several sectors also show signs of saturation, including agriculture under the pressure of large demographic growth, demonstrating crop yield stagnation (IEA, 2016), soil overexploitation, and perturbations of ecological processes leading to large losses in biodiversity (Cardinale et al., 2012; Maxwell et al., 2016). Regarding the complex interactions between organisms and their milieu, enhanced environmental constraints and reduced primary resources at the same time may be the simultaneous keys for predicting future human performances (Rockström et al., 2009; Carnes et al., 2014; Finch et al., 2014).

\section{TO THE UPPER LIMITS}

\section{Physical Performances}

"There is a vast store of accurate information, hitherto almost unexploited, in the records of athletic sports and racing" (Hill,
1925). Since the pioneering work of A.V. Hill, sport performances have provided valuable data to grasp human physiology, as they represent the most accurate measurements of human potentials and capabilities (Hill, 1925; Larry et al., 2012; Hawley et al., 2014; Berthelot et al., 2015). Their historical trends provide precise ways of measuring human progress, its consecutive steps and determinants.

As no one has been able to surpass their performance, record holding athletes express the limits of human physiology (Hill, 1925; Norton and Olds, 2001; Berthelot et al., 2015). Yearly maximal performances also represent a unique biomarker for the understanding of age-related changes across lifespan at both individual and population levels, providing a simple phenotypic indicator of growth and aging (Berthelot et al., 2011; Larry et al., 2012; Justice et al., 2016; Marck et al., 2016). Finally, maximal performance also appears as a main indicator of the relationship linking physiology and health to environmental changes (for example, the impacts of temperature changes) (El Helou et al., 2012; Larry et al., 2012; Haïda et al., 2013).

The analysis of historical sport achievements offers an accurate and original reading of our society's progressions and accelerations during the twentieth century (Guillaume et al., 2009). Following the Olympic motto "citius, altius, fortius," best performances in all metric and chronometric sports including running (Nevill and Whyte, 2005; Berthelot et al., 2008, 2010b, 2015; Denny, 2008), swimming (Nevill et al., 2007; Berthelot et al., 2008, 2010a, 2015), jumping (Berthelot et al., 2008, 2010a, 2015), weightlifting (Berthelot et al., 2008), cycling (Desgorces et al., 2008; El Helou et al., 2010), skiing (Desgorces et al., 2008), or skating (Kuper and Sterken, 2003; Berthelot et al., 2008) considerably progressed, until the end of the twentieth century, except during World Wars I and II (Figures 1A,B).

In the marathon, the best time by men decreased from the first world best performance ratified by the IAAF (International Association of Athletics Federations) in 1908 ( $2 \mathrm{~h} 55 \mathrm{~min} 18 \mathrm{~s}$ ) to the current world best performance ( $2 \mathrm{~h} 02 \mathrm{~min} 57 \mathrm{~s}$ ). Likewise, in the $100 \mathrm{~m}$ swimming free style the world record progressed from $65 \mathrm{~s}$ in 1905 to $49.91 \mathrm{~s}$ in 2009 , while the hour cycling record increased from $26.508 \mathrm{~km}$ in 1876 to $54.526 \mathrm{~km}$ in 2015 .

Can we forecast future world record evolution in sports? Recent data have shown a common slow-down in both frequency and increments for all the Olympic chronometric and metric events (Berthelot et al., 2008, 2010b). Historical series from 1896 to 2016 in track and field, swimming, cycling, skating, and weight-lifting reveal a similar pattern suggesting a trend toward a plateau during the last three decades for both sexes (Blest, 1996; Reinboud, 2004; Berthelot et al., 2008, 2010b, 2015; Denny, 2008; Haugen et al., 2015) (Figures 1A,B). In fact, despite an average lag of 30 years between the beginning of competition for men and women, performances by women also began to plateau since the mid-1980s (Thibault et al., 2010; Berthelot et al., 2015).

In the absence of future changes in rules or technological improvements, plateaus obtained after an asymptotic progression may now indicate the potential upper limits of $H$. sapiens. This was suggested by former biomechanical and physiological investigations including structural and functional factors such as limiting oxygen uptake, maximal heart rate, muscle mass 

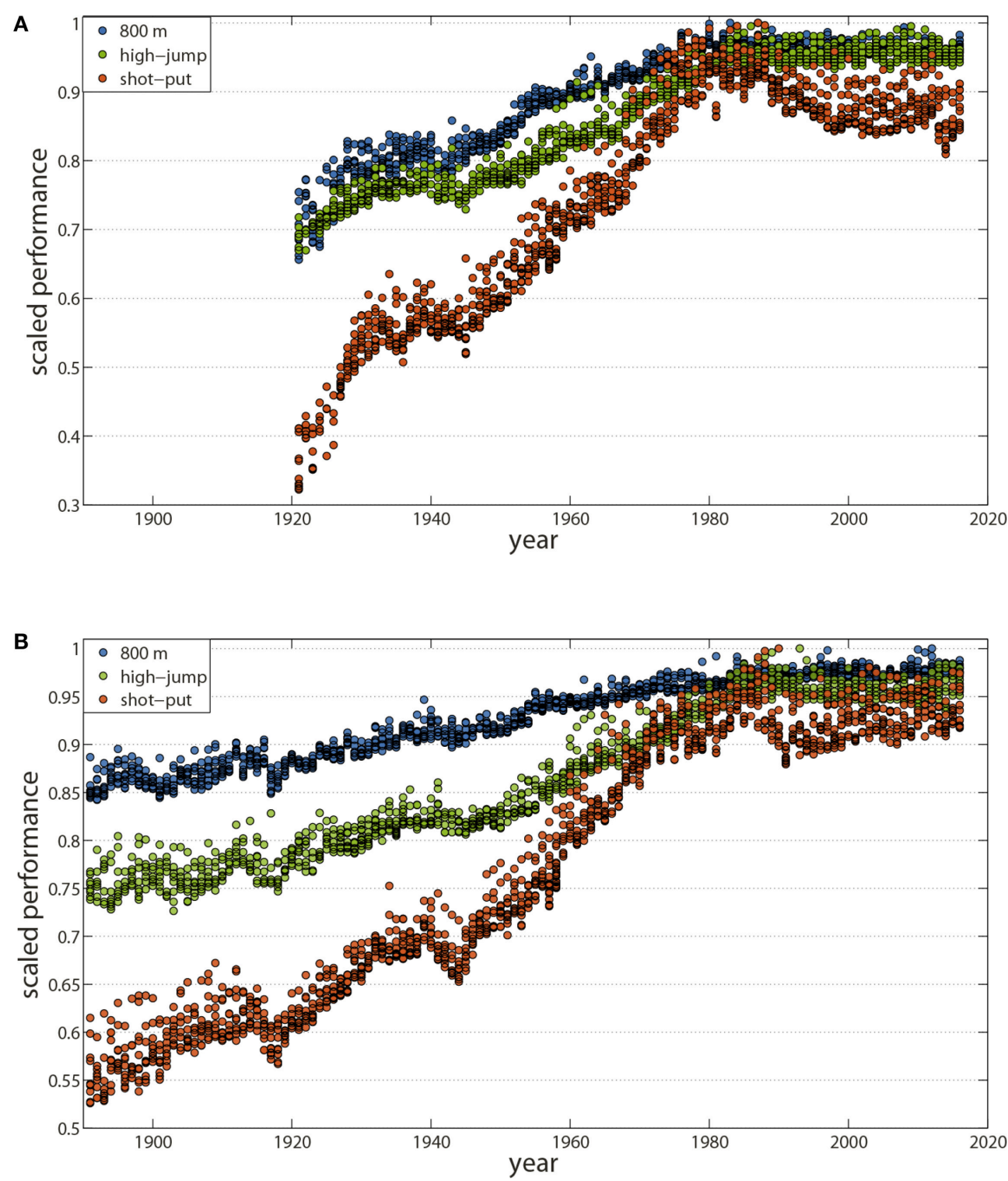

FIGURE 1 | Maximal physical performance has plateaued during the last three decades. Historical series of the best performances in Track and Field events have shown trends of plateauing since 1980 for both men and women, one of the early signs of established human physiological limits. (A) Annual World's 10 best performances from 1921 to 2016 in women for the 800 m event (blue), the high-jump event (green), and the shot-put (orange). (B) Annual World's 10 best performances from 1896 to 2016 in men for the $800 \mathrm{~m}$ event (blue), the high-jump event (green), and the shot-put (orange). Data are from International Association of Athletics Federations (IAAF: https://www.iaaf.org/home) and are normalized by the respective world records for the event at the end of 2016, that is, performances are divided by the current world record.

and contraction, reaction time, stature, and stride length and frequency (Bennett, 1989; Bassett and Howley, 2000; Weyand et al., 2010; Ferretti et al., 2011; Berthelot et al., 2015; Haugen et al., 2015). Moreover, recent trends in track and field (e.g., regression in Throws and Jumps) suggest that limits have already been artificially enhanced through doping practices (Spedding and Spedding, 2008; Berthelot et al., 2015). Pharmacological innovations and the Cold War exacerbated the use of performance-enhancing drugs including EPOs, growth hormones, steroid hormones, or amphetamines (Franke and
Berendonk, 1997; Spedding and Spedding, 2008; Guillaume et al., 2009; Berthelot et al., 2010a, 2015). Their effects on elite athletes are difficult to precisely measure, but are certainly related to the last "burst" of performance during the eighties and nineties (Spedding and Spedding, 2008; Berthelot et al., 2010a, 2015; El Helou et al., 2010; Durussel et al., 2013). Also, technology is a main source of potential enhancement. The three successive generation of suits over the 1990-2009 period improved world swimmers' performance by a mean of 3\% before their ban in 2010 (Berthelot et al., 2010a, 2015). 
In the general population, long historical series in Western Europe or Scandinavian countries shows a decline in endurance and strength performance of young men (Rasmussen et al., 1999; Santtila et al., 2006; Tomkinson, 2007; Huotari et al., 2010; Runhaar et al., 2010). Studies indicate that fewer individuals reach excellent physical performance, while more and more subjects remain at low physical performance levels (Santtila et al., 2006; Huotari et al., 2010). Regression in fitness capacity may be due to reduced physical activity in most developed societies (Tomkinson, 2007; Tomkinson and Olds, 2007; Tomkinson et al., 2012; Lang et al., 2016).

Time series in sports are certainly one of the most relevant evidences in the understanding of human upper limits. They not only assess the synergistic process of human improvement until its full optimization, but also relate to the finite body of each athlete with her or his absolute limits at each spatial and temporal scale.

\section{Lifespan}

Human life-expectancy and maximal lifespan trends also provide long historical series (Olshansky et al., 1990; Oeppen and Vaupel, 2002; Le Bourg, 2012; da Silva Antero-Jacquemin et al., 2014; Dong et al., 2016). Similar to sport achievements, though somewhat less precisely measured, it followed an unprecedented progression during the twentieth century supported by major nutritional, scientific, technological, societal, and medical innovations (Wilmoth, 2000; Oeppen and Vaupel, 2002; Fogel, 2004; Omran, 2005; Olshansky and Carnes, 2009; Le Bourg, 2012; da Silva Antero-Jacquemin et al., 2014). From 1900 to 2000 in the majority of high-income countries, life expectancy at birth increased by $\sim 30$ years (Wilmoth, 2000; Hayflick, 2007), mostly due to a reduction of child mortality through nutrition, hygiene, vaccination, and other medical improvements (Wilmoth, 2000; Fogel, 2004; Omran, 2005; Hayflick, 2007).

Concerning the future, trends oscillate, from pessimistic to optimistic views (Vaupel, 1997; Wilmoth, 2000; Oeppen and Vaupel, 2002; Christensen et al., 2009; Olshansky and Carnes, 2009; Le Bourg, 2012; da Silva Antero-Jacquemin et al., 2014; Dong et al., 2016; Gavrilov et al., 2017; Kirkwood, 2017; Le Bourg and Vijg, 2017; Vijg and Le Bourg, 2017), but recent data suggest a slow-down in the progress of life-expectancy related to the stabilization of a very low level of infant mortality $(0.2-1 \%$ of births in the healthiest countries in the world) (Wilmoth, 2000). The present slow progress in high-income countries is mostly due to reduced mortality rates of chronic non-communicable diseases, principally among cardiovascular diseases and cancers. However, those advancements have a much lower impact on lifeexpectancy as compared to vaccination campaigns (Wilmoth, 2000; Olshansky et al., 2001; Buchanan, 2016). In these countries, the last two decades showed a slow-down and even a reduction of life-expectancy in specific populations, such as the EuroAmerican women in the USA, that may indicate the first change in life-expectancy trends (Case and Deaton, 2015; Shiels, 2017).

Predicting a continuous linear growth of life-expectancy in the long term may probably not be relevant if the major progresses have already been accomplished. Beyond the fittest mathematical model for estimating future trends, we need to carefully examine the consistency with structural and functional limits determining maximal lifespan related to life-history strategies and evolutionary and environmental constraints (Carnes and Olshansky, 2007; Hayflick, 2007; Olshansky and Carnes, 2009; Le Bourg, 2012; Le Bourg and Vijg, 2017; Vijg and Le Bourg, 2017). For example, aging is an irreversible process: it is complex as it concerns all physiological functions, organs, and maintenance systems. But, it also has universal characteristics, showing a continuous exponential decline starting in the third decade for all maximal indicators with an accelerated loss of physical performance until death (Moore, 1975; Berthelot et al., 2011; Marck et al., 2016). Despite the recent phenotypic expansion, such a widespread dynamic remained, showing no escape from decline, despite the best efforts of the oldest old (Moore, 1975; Berthelot et al., 2011; Marck et al., 2016).

Similarly, maximal lifespan increased slightly during the last two centuries (Robine and Vaupel, 2002), but since 1997, nobody has lived for more than 120 years. Surpassing mathematical models, projecting 300 years in the future without biological considerations, most recent data showed evidence of a lifespan plateau around 115-120 years (Le Bourg, 2012; da Silva AnteroJacquemin et al., 2014; Dong et al., 2016), despite a sharp increase in the number of centenarians and supercentenarians (Figure 2). Jeanne Calment with 122.4 years has certainly come close to the potential biological limit of our species in term of longevity, at the benefit of an extremely rare long-lived phenotype supported by a specific lifestyle and chance (Dong et al., 2016).

Olympian and elite athletes also constitute a highly selected population with a 6-7 years longer life duration as compared to the general population (Marijon et al., 2013; Antero-Jacquemin et al., 2015). With a maximum of 106 years, maximal lifespan in this population paralleled the supercentenarian trend (da Silva Antero-Jacquemin et al., 2014). Both populations indicate a similar densification beyond the upper limit: more and more individuals reach values close to the limit, but do not surpass it, resulting in a progressive rectangularization of their survival curve (Kannisto, 2000; Andersen et al., 2012; da Silva AnteroJacquemin et al., 2014).

\section{Adult Height}

Human height is another simple and accurate biomarker integrating both environmental and nutritional conditions encountered during fetal development and childhood (Fogel, 2004; Komlos and Baur, 2004; NCD-RisC, 2016; Stulp and Barrett, 2016). The recent study by the NCD Risk Factor Collaboration offers a large overview of secular trends in adult height for most countries in the world (NCD-RisC, 2016). The average gain in adult height for cohorts born between 1896 and 1996 was estimated at $8.3 \pm 3.6 \mathrm{~cm}$ for women and $8.8 \pm 3.5 \mathrm{~cm}$ for men (Larnkjær et al., 2006; Baten and Blum, 2012; Schönbeck et al., 2013; NCD-RisC, 2016; Stulp and Barrett, 2016).

The analysis of growth patterns within world regions reveals that the gain in height during the last century was not a linear process (Komlos and Baur, 2004; Larnkjær et al., 2006; Komlos and Lauderdale, 2007; Rashad, 2008; Komlos, 2010; Baten and Blum, 2012; Schönbeck et al., 2013; NCD-RisC, 2016) (Figures 3A,B). During the last three decades, data have shown 


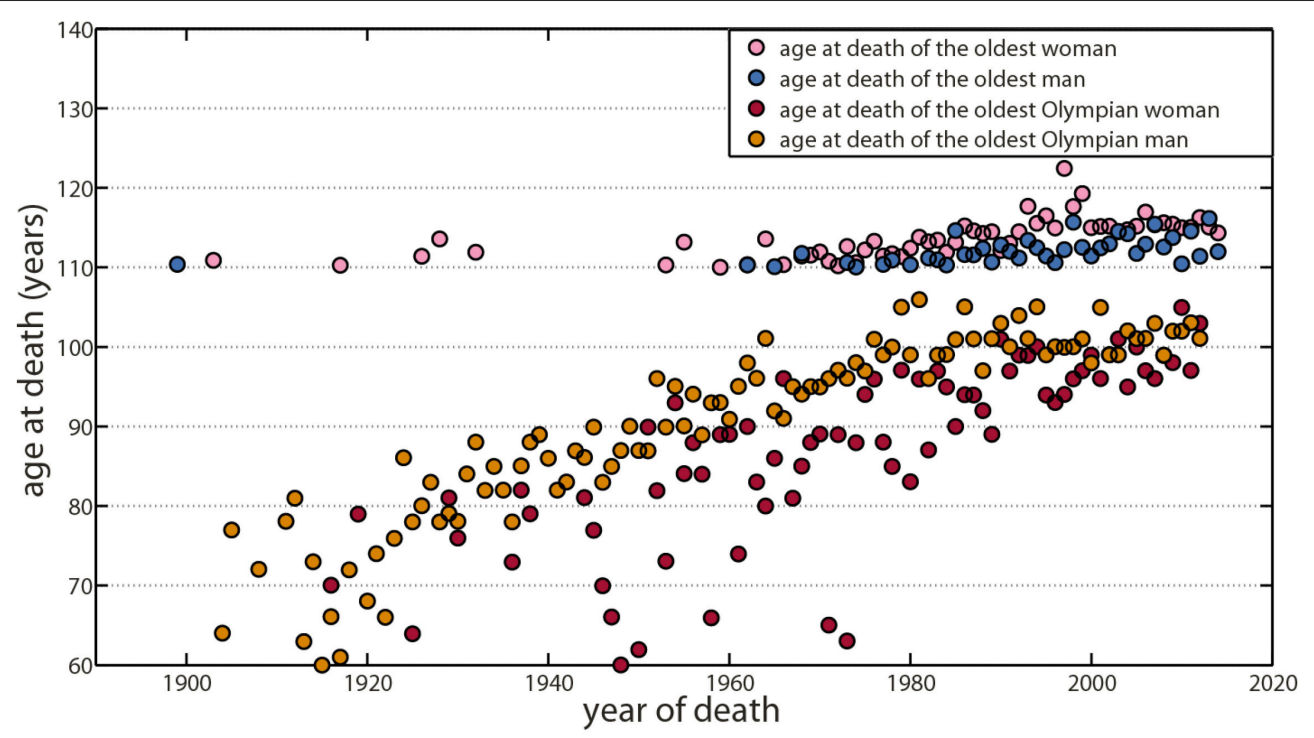

FIGURE 2 | Maximal lifespan trends have shown a plateau for the oldest supercentenarian and the oldest Olympian. Since Jeanne Calment, who currently holds the lifespan record at 122.4 years in 1997, no one has lived more than 120 years and data suggest a plateau around 115-117 years that may indicate the potential biological upper limit of our species in terms of longevity. Oldest supercentenarian trends have shown a plateau for both women (purple) and men (blue). Similarly, maximal lifespan trends for oldest Olympians have shown a plateau for both women (red) and men (orange). Data for supercentenarians are available at the Gerontological Research Group (GRG; http://www.grg.org/). Data for Olympian athletes came from the most authoritative source of Olympian biographies (Clarke et al., 2012; Antero-Jacquemin et al., 2015).

a similar plateau in the tallest populations among high-income countries from North America to Europe (Komlos and Baur, 2004; Larnkjær et al., 2006; Komlos and Lauderdale, 2007; Rashad, 2008; Komlos, 2010; Schönbeck et al., 2013; NCDRisC, 2016; Stulp and Barrett, 2016) (Figures 3A,B). This recent asymptote suggests, we are approaching another of our upper limits (Le Bourg, 2012; NCD-RisC, 2016).

Such a trend was also observed among elite athletes (Norton and Olds, 2001; Sedeaud et al., 2014). After a large gain in the mid-twentieth century, a plateau has been described for all major US sports, which select especially tall individuals (Sedeaud et al., 2014). For example, in the NFL (National Football League) from 1920 to 2010 , average players' height gained $8.1 \mathrm{~cm}(0.9$ $\mathrm{cm} /$ decade, from 179.6 to $187.7 \mathrm{~cm}$ ). However, during the last 30 years, male height has plateaued at $187 \mathrm{~cm}$ (Sedeaud et al., 2014). The NBA (National Basketball Association) evidenced the most obvious ceiling (Sedeaud et al., 2014): about $200 \mathrm{~cm}$, since 1984. NHL, MLB (Major league Baseball), NFL and NBA height evolutions suggest a similar common trend linked to the best metric and chronometric performances among highly selected athletes (Sedeaud et al., 2014; Berthelot et al., 2015).

\section{WHAT ARE THE IMPLICATIONS?}

For millennia, it was difficult to test whether physiological limits do exist in humans, because no variation was accurately measurable. It is now possible to ascertain the biological limits of the human species, through sport records, lifespan, or height. These traits no longer increase, despite further continuous nutritional, medical, and scientific progress. This suggests that modern societies have allowed our species to reach its limits. We are the first generation to become aware of these limitations.

Regarding sport records, the first consequence is that increasingly less records will be broken in the coming years (Berthelot et al., 2008, 2010b). Humans need perspectives and, for this reason, in an attempt to reactivate interest in athletic progress, it was already proposed to start a new series of records or to change event rules (Spedding and Spedding, 2008; Berthelot et al., 2015). In this respect, the $2 \mathrm{~h}$ and $25 \mathrm{~s}$ marathon ran by Eliud Kipchoge for an exhibition in Italy has shown how improvement can be obtained under totally optimized and artificial conditions. Such improvement will substantially and artificially enhance maximal performance without reconsidering the general idea of existing upper biological limits.

In fact, it is probable that human natural limits have already been enhanced by artificial means for both maximal longevity and maximal physical performance. These "manufactured times", represent an increase in life duration or sport performance, beyond the limits imposed by our biology (Carnes and Olshansky, 2007). In this sense, a scientific breakthrough may point to another future substantial "manufactured" gain that will shift the upper limits beyond the current values in addition to the healthy living standards that will increase the number of people reaching old age. However, such artificial enhancements will also have "Achilles heels", i.e., maximal progress that cannot surpass an imposed ceiling. For example, the evolutionary constraints of body design that lead to structural and functional limitations or environmental factors hinder increased progress. The emergence of new major artificial enhancements may 

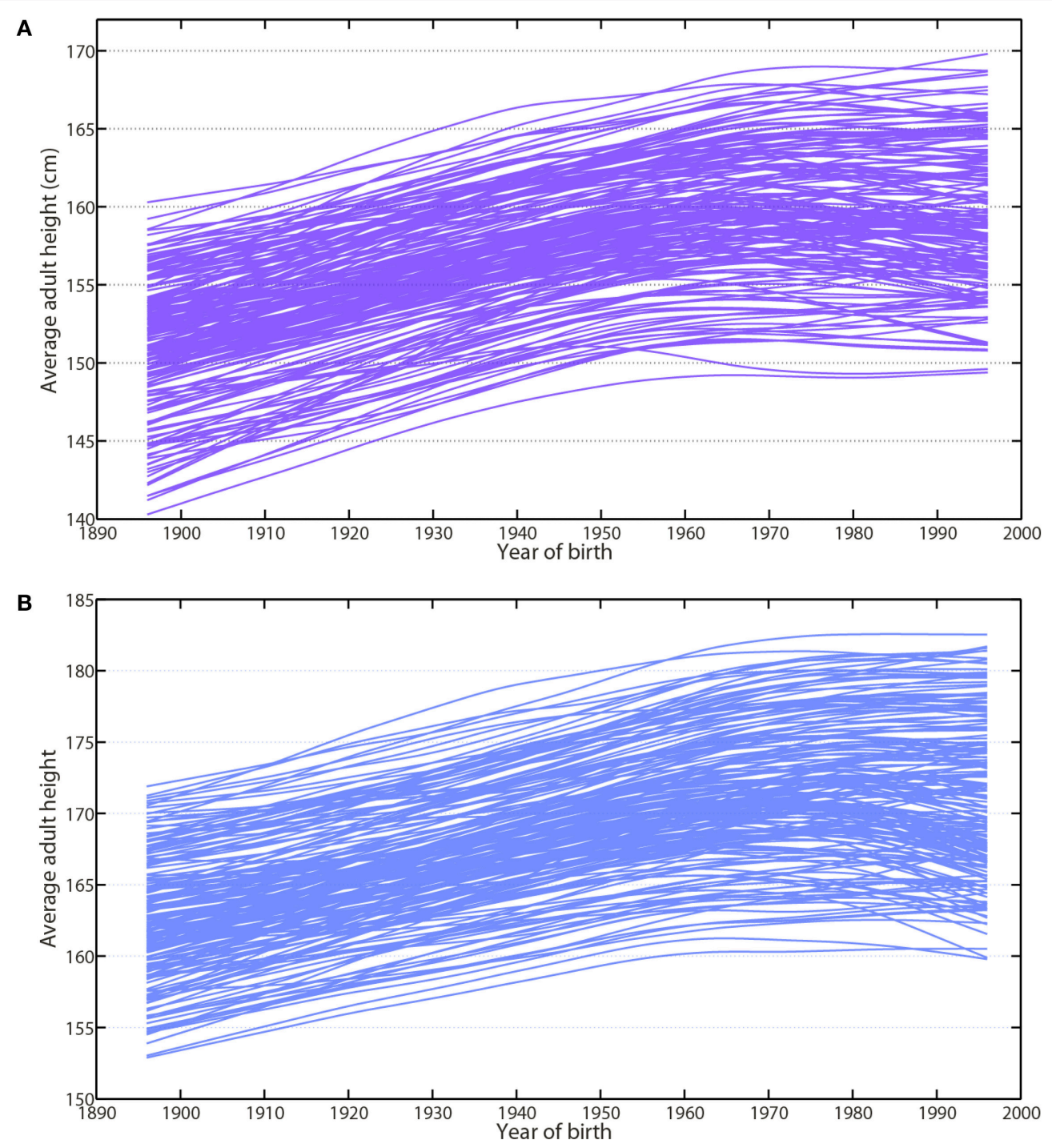

FIGURE 3 | Average adult height has followed a similar pattern toward a plateauing trend since 1980. The analysis of the growth patterns reveals that the gain in height during the last century is not a linear process. During the last 3 decades, data have shown a similar plateauing state in the tallest populations among women and men of high-income countries from North America to Europe. This recent asymptote suggests a potential upper limit to human height. (A) Average adult height of women (violet-purple) represented for each country from 1914 up to 2014. (B) Average adult height of men (blue) represented for each country from 1914 up to 2014. Data are compiled from NCD-RisC and available by country on their website (https://elifesciences.org/content/5/e13410).

be less favorable in light of ever increasing environmental boundaries.

For such reasons, it is meaningless to claim that most human will live for 200-500 years in the near future (de Grey, 2003), thanks to medical or scientific progress, or that "within 15 years, we'll be adding more than a year every year to our remaining life expectancy" (Kurzweil and Grossman, 2010). Raising false hopes without taking into account that human beings are already extremely "optimized" for lifespan seems inappropriate.

In the scenario of limited performance, the interest on strategies for increasing quality of life reaches greater importance, such as investing in grassroots sports in order to enhance health (European Commission, 2016). If a country is able to promote human development and health, one should observe an incremental rise in values of mean adult height, sport performance, and lifespan. The utmost challenge is to maintain these indices at high values. Under escalating environmental constraints, this may cost increasingly more energy and investment to balance the rising ecosystemic pressures in order to maintain our performance levels. Indeed observing decreasing tendencies may provide an early signal that something has changed but not for the better. Human height has decreased in the last decade in some African countries; this suggests some societies are no longer able to provide sufficient nutrition for each of their children and maintain the health of their younger inhabitants (NCD-RisC, 2016). 
Knowing limits of the human species indicates clear goals for any nation; states should govern in such a way that human size, lifespan, and physical performance increase in order to reach their highest values for most people. When plateaus are reached, care should then be taken to prevent regression even if remaining close to the upper limits may become more costly. This aim will be one of the most intense challenges of this century, especially with the new pressure of anthropocenic activities responsible for deleterious effects on both humans, environment and health (Rockström et al., 2009; Steffen et al., 2011, 2015; Carnes et al., 2014; Finch et al., 2014; McMichael, 2014). However, solutions may be found and collective actions may taken to restrain this pressure and, in an optimistic way, maintain the possibility of reaching and remaining at the upper limits(United Nations, 2016).

\section{REFERENCES}

Aarssen, K., and de Haan, L. (1994). On the maximal life span of humans. Math. Popul. Stud. 4, 259-281. doi: 10.1080/08898489409525379

Andersen, S. L., Sebastiani, P., Dworkis, D. A., Feldman, L., and Perls, T. T. (2012). Health span approximates life span among many supercentenarians: compression of morbidity at the approximate limit of life span. J. Gerontol. Ser. A 67A, 395-405. doi: 10.1093/gerona/glr223

Antero-Jacquemin, J., Rey, G., Marc, A., Dor, F., Haïda, A., Marck, A., et al. (2015). Mortality in female and male french olympians: a 1948-2013 cohort study. Am. J. Sports Med. 43, 1505-1512. doi: 10.1177/03635465155 74691

Bassett, D. R., and Howley, E. T. (2000). Limiting factors for maximum oxygen uptake and determinants of endurance performance. Med. Sci. Sports Exerc. 32, 70-84. doi: 10.1097/00005768-200001000-00012

Baten, J., and Blum, M. (2012). Growing tall but unequal: new findings and new background evidence on anthropometric welfare in 156 countries, 1810-1989. Econ. Hist. Dev. Reg. 27, S66-S85. doi: 10.1080/20780389.2012.657489

Beltrán-Sánchez, H., Crimmins, E. M., and Finch, C. E. (2012). Early cohort mortality predicts the rate of aging in the cohort: a historical analysis. J. Dev. Orig. Health Dis. 3, 380-386. doi: 10.1017/S2040174412000281

Bennett, A. (1989). "Integrated studies of locomotor performance," in Complex Organismal Functions: Integration and Evolution in Vertebrates, eds D.B. Wake and G. Roth (Chichester: John Wiley and Sons), 191-202.

Berthelot, G., Len, S., Hellard, P., Tafflet, M., El Helou, N., Escolano, S., et al. (2010a). Technology \& swimming: 3 steps beyond physiology. Mater. Today 13, 46-51. doi: 10.1016/S1369-7021(10)70203-0

Berthelot, G., Len, S., Hellard, P., Tafflet, M., Guillaume, M., Vollmer, J. C., et al. (2011). Exponential growth combined with exponential decline explains lifetime performance evolution in individual and human species. AGE 34, 1001-1009. doi: 10.1007/s11357-011-9274-9

Berthelot, G., Sedeaud, A., Marck, A., Antero-Jacquemin, J., Schipman, J., Saulière, G., et al. (2015). Has athletic performance reached its peak? Sports Med. 45, 1263-1271. doi: 10.1007/s40279-015-0347-2

Berthelot, G., Tafflet, M., El Helou, N., Len, S., Escolano, S., Guillaume, M., et al. (2010b). Athlete atypicity on the edge of human achievement: performances stagnate after the last peak in 1988. PLoS ONE 5:e8800 doi: 10.1371/journal.pone.0008800

Berthelot, G., Thibault, V., Tafflet, M., Escolano, S., El Helou, N., Jouven, $\mathrm{X}$, et al. (2008). The citius end: world records progression announces the completion of a brief ultra-physiological quest. PLOS ONE 3:e1552 doi: 10.1371/journal.pone.0001552

Bianconi, E., Piovesan, A., Facchin, F., Beraudi, A., Casadei, R., Frabetti, F., et al. (2013). An estimation of the number of cells in the human body. Ann. Hum. Biol. 40, 463-471. doi: 10.3109/03014460.2013.807878

Blest, D. C. (1996). Lower bounds for athletic performance. J. R. Stat. Soc. Ser. Stat. 45, 243-253. doi: $10.2307 / 2988413$

\section{AUTHOR CONTRIBUTIONS}

AM, JA, GS, and GeB collected the data. AM, JA, GS, and GeB analyzed them. AM, JA, GeB, VMD, $\mathrm{GiB}$, MS, ELB, and JFT wrote the paper and all authors contributed to the scientific debate. JFT is the guarantor.

\section{ACKNOWLEDGMENTS}

The authors thank Stacey Johnson, Jean-Marc Di Meglio, Tom Wyatt, Gaëlle Woestelandt, Damien Marck, and Patrick Marck for their advice and careful reading. We also thank Gregoire Millet and the two reviewers for their advice and rigorous comments.

Blomberg, S. P., and Garland, T. (2002). Tempo and mode in evolution: phylogenetic inertia, adaptation and comparative methods. J. Evol. Biol. 15, 899-910. doi: 10.1046/j.1420-9101.2002.00472.x

Bohacek, J., and Mansuy, I. M. (2015). Molecular insights into transgenerational non-genetic inheritance of acquired behaviours. Nat. Rev. Genet. 16, 641-652. doi: $10.1038 / \mathrm{nrg} 3964$

Bramble, D. M., and Lieberman, D. E. (2004). Endurance running and the evolution of Homo. Nature 432, 345-352. doi: 10.1038/nature03052

Bravo, J., and Real, P. (2012). Modeling Longevity Risk Using Extreme Value Theory: An Empirical Investigation Using Portuguese and Spanish Population Data. Technical report, Evora University, Portugal.

Brown, N. J. L., Albers, C. J., and Ritchie, S. J. (2017). Contesting the evidence for limited human lifespan. Nature 546, E6-E7. doi: 10.1038/nature22784

Buchanan, M. (2016). Lifespan limits. Nat. Phys. 12, 989-989. doi: $10.1038 /$ nphys3951

Campisi, J. (1996). Replicative senescence: an old lives' tale? Cell 84, 497-500. doi: 10.1016/S0092-8674(00)81023-5

Cardinale, B. J., Duffy, J. E., Gonzalez, A., Hooper, D. U., Perrings, C., Venail, P., et al. (2012). Biodiversity loss and its impact on humanity. Nature 486, 59-67. doi: $10.1038 /$ nature 11148

Carnes, B. A., and Olshansky, S. J. (2007). A realist view of aging, mortality, and future longevity. Popul. Dev. Rev. 33, 367-381. doi: 10.1111/j.1728-4457.2007.00172.x

Carnes, B. A., Staats, D., and Willcox, B. J. (2014). Impact of climate change on elder health. J. Gerontol. Ser. A 69, 1087-1091. doi: 10.1093/gerona/glt159

Case, A., and Deaton, A. (2015). Rising morbidity and mortality in midlife among white non-Hispanic Americans in the 21st century. Proc. Natl. Acad. Sci. U.S.A. 112, 15078-15083 doi: 10.1073/pnas.15183 93112

Charlesworth, B. (2009). Fundamental concepts in genetics: effective population size and patterns of molecular evolution and variation. Nat. Rev. Genet. 10, 195-205. doi: 10.1038/nrg2526

Chatterjee, S., and Chatterjee, S. (1982). New lamps for old: an exploratory analysis of running times in olympic games. J. R. Stat. Soc. Ser. C Appl. Stat. 31, 14-22. doi: $10.2307 / 2347070$

Christensen, K., Doblhammer, G., Rau, R., and Vaupel, J. W. (2009). Ageing populations: the challenges ahead. Lancet 374, 1196-1208. doi: 10.1016/S0140-6736(09)61460-4

Clarke, P. M., Walter, S. J., Hayen, A., Mallon, W. J., Heijmans J., and Studdert, D. M. (2012). Survival of the fittest: retrospective cohort study of the longevity of Olympic medallists in the modern era. BMJ 345:e8308. doi: 10.1136/bmj.e8308

Couzin-Frankel, J. (2011). A pitched battle over life span. Science 333, 549-550. doi: 10.1126/science.333.6042.549

da Silva Antero-Jacquemin, J., Berthelot, G, Marck, A, Noirez, P, Latouche, A, and Toussaint, J. F. (2014). Learning from leaders: life-span trends in olympians and supercentenarians. J. Gerontol. A. Biol. Sci. Med. Sci. 70, 944-949. doi: 10.1093/gerona/glu130 
de Beer, J., Bardoutsos, A., and Janssen, F. (2017). Maximum human lifespan may increase to 125 years. Nature 546, E16-E17. doi: 10.1038/nature22792

de Grey, A. (2003). "Foreseeable and more distant rejuvenation therapies," in Aging Interventions and Therapies, ed S. I. S. Rattan (Singapore: World Scientific Publishers), 379-395.

Deakin, M. (1967). Estimating bounds on athletic performance. Math. Gaz. 51, 100-103. doi: $10.2307 / 3614380$

Denny, M. W. (2008). Limits to running speed in dogs, horses and humans. J. Exp. Biol. 211, 3836-3849. doi: 10.1242/jeb.024968

Desgorces, F. D., Berthelot, G., Charmantier, A., Tafflet, M., Schaal, K., Jarne, P., et al. (2012). Similar slow down in running speed progression in species under human pressure. J. Evol. Biol. 25, 1792-1799. doi: 10.1111/j.1420-9101.2012.02563.x

Desgorces, F. D., Berthelot, G., El Helou, N., Thibault, V., Guillaume, M., Tafflet, M., et al. (2008). From oxford to hawaii ecophysiological barriers limit human progression in ten sport monuments. PLOS ONE 3:e3653 doi: 10.1371/journal.pone.0003653

DeWitt, T. J., Sih, A., and Wilson, D. S. (1998). Costs and limits of phenotypic plasticity. Trends Ecol. Evol. 13, 77-81. doi: 10.1016/S0169-5347(97)01274-3

Dhar, P. K., and Giuliani, A. (2010). Laws of biology: why so few? Syst. Synth. Biol. 4, 7-13. doi: 10.1007/s11693-009-9049-0

Domínguez-Rodrigo, M., Pickering, T. R., and Bunn, H. T. (2010). Configurational approach to identifying the earliest hominin butchers. Proc. Natl. Acad. Sci. U.S.A. 107, 20929-20934. doi: 10.1073/pnas.1013711107

Dong, X., Milholland, B., and Vijg, J. (2016). Evidence for a limit to human lifespan. Nature 538, 257-259. doi: 10.1038/nature19793

Durussel, J., Daskalaki, E., Anderson, M., Chatterji, T., Wondimu, D. H., Padmanabhan, N., et al. (2013). Haemoglobin mass and running time trial performance after recombinant human erythropoietin administration in trained men. PLOS ONE 8:e56151. doi: 10.1371/journal.pone.0056151

El Helou, N., Berthelot, G., Thibault, V., Tafflet, M., Nassif, H., Campion, F., et al. (2010). Tour de France, Giro, Vuelta, and classic European races show a unique progression of road cycling speed in the last 20 years. J. Sports Sci. 28, 789-796. doi: $10.1080 / 02640411003739654$

El Helou, N., Tafflet, M., Berthelot, G., Tolaini, J., Marc, A., et al. (2012). Impact of environmental parameters on marathon running performance. PLoS ONE 7:e37407. doi: 10.1371/journal.pone.0037407

European Commission (2016). On the Implementation of the Council Recommendation on Promoting Health-Enhancing Physical Activity Across Sectors. Brussels: European Commission.

Ezkurdia, I., Juan, D., Rodriguez, J. M., Frankish, A., Diekhans, M., Harrow, J., et al. (2014). Multiple evidence strands suggest that there may be as few as 19000 human protein-coding genes. Hum. Mol. Genet. 23, 5866-5878. doi: $10.1093 / \mathrm{hmg} / \mathrm{ddu} 309$

Ferretti, G., Bringard, A., and Perini, R. (2011). An analysis of performance in human locomotion. Eur. J. Appl. Physiol. 111, 391-401. doi: 10.1007/s00421-010-1482-y

Finch, C. E., Beltrán-Sánchez, H., and Crimmins, E. M. (2014). Uneven futures of human lifespans: reckonings from gompertz mortality rates, climate change, and air pollution. Gerontology 60, 183-188. doi: 10.1159/000357672

Fogel, R. W. (2004). The Escape from Hunger and Premature Death, 1700-2100: Europe, America, and the Third World. New York, NY: Cambridge University Press.

Foster, L., James, D., and Haake, S. (2010). Understanding the influence of population size on athletic performance. Proced. Eng. 2, 3183-3189. doi: $10.1016 / j$.proeng.2010.04.130

Franke, W. W., and Berendonk, B. (1997). Hormonal doping and androgenization of athletes: a secret program of the German Democratic Republic government. Clin. Chem. 43, 1262-1279.

Fumagalli, M., Moltke, I., Grarup, N., Racimo, F., Bjerregaard, P., Jørgensen, M. E., et al. (2015). Greenlandic Inuit show genetic signatures of diet and climate adaptation. Science 349, 1343-1347. doi: 10.1126/science.aab2319

Gapp, K., von Ziegler, L., Tweedie-Cullen, R. Y., and Mansuy, I. M. (2014). Early life epigenetic programming and transmission of stress-induced traits in mammals: how and when can environmental factors influence traits and their transgenerational inheritance? BioEssays News Rev. Mol. Cell. Dev. Biol. 36, 491-502. doi: 10.1002/bies.201300116
Gavrilov, L. A., Krut'ko, V. N., and Gavrilova, N. S. (2017). The future of human longevity. Gerontology 63, 524-526. doi: 10.1159/000477965

Gbari, S., Poulain, M., Dal, L., and Denuit, M. (2017). Extreme value analysis of mortality at the oldest ages: a case study based on individual ages at death. North Am. Actuar. J. 21, 397-416. doi: 10.1080/10920277.2017.1301260

Gillespie, J. H. (2001). Is the population size of a species relevant to its evolution? Evolution 55, 2161-2169. doi: 10.1111/j.0014-3820.2001.tb00732.x

Grigaltchik, V. S., Ward, A. J. W., and Seebacher, F. (2012). Thermal acclimation of interactions: differential responses to temperature change alter predator-prey relationship. Proc. R. Soc. Lond. B Biol. Sci. 279, 4058-4064. doi: 10.1098/rspb.2012.1277

Guillaume, M., Helou, N. E., Nassif, H., Berthelot, G., Len, S., Thibault, V., et al. (2009). Success in developing regions: world records evolution through a geopolitical prism. PLoS ONE 4:e7573. doi: 10.1371/journal.pone.0007573

Haake, S. J., Foster, L. I., and James, D. M. (2014). An improvement index to quantify the evolution of performance in running. J. Sports Sci. 32, 610-622. doi: 10.1080/02640414.2013.841974

Haïda, A., Dor, F., Guillaume, M., Quinquis, L., Marc, A., Marquet, L. A., et al. (2013). Environment and scheduling effects on sprint and middle distance running performances. PLoS ONE 8:e79548. doi: 10.1371/journal.pone.0079548

Hanayama, N., and Sibuya, M. (2016). Estimating the upper limit of lifetime probability distribution, based on data of japanese centenarians. J. Gerontol. Ser. A 71, 1014-1021. doi: 10.1093/gerona/glv113

Haugen, T., Tønnessen, E., and Seiler, S. (2015). 9.58 and 10.49: nearing the citius end for $100 \mathrm{~m}$ ? Int. J. Sports Physiol. Perform. 10, 269-272. doi: 10.1123/ijspp.2014-0350

Hawley, J. A., Hargreaves, M., Joyner, M. J., and Zierath, J. R. (2014). Integrative biology of exercise. Cell 159, 738-749. doi: 10.1016/j.cell.2014.10.029

Hayflick, L. (1965). The limited in vitro lifetime of human diploid cell strains. Exp. Cell Res. 37, 614-636. doi: 10.1016/0014-4827(65)90211-9

Hayflick, L. (2007). Biological aging is no longer an unsolved problem. Ann. N. Y. Acad. Sci. 1100, 1-13. doi: 10.1196/annals.1395.001

Hill, A. V. (1925). The physiological basis of athletic records. Lancet 206, 481-486. doi: 10.1016/S0140-6736(01)15546-7

Holstege, H., Pfeiffer, W., Sie, D., Hulsman, M., Nicholas, T. J., Lee, C. C., et al. (2014). Somatic mutations found in the healthy blood compartment of a 115-yr-old woman demonstrate oligoclonal hematopoiesis. Genome Res. 24, 733-742. doi: 10.1101/gr.162131.113

Hublin, J.-J., Neubauer, S., and Gunz, P. (2015). Brain ontogeny and life history in Pleistocene hominins. Phil. Trans. R. Soc. B 370:20140062. doi: 10.1098/rstb.2014.0062

Hughes, B. G., and Hekimi, S. (2017). Many possible maximum lifespan trajectories. Nature 546, E8-E9. doi: 10.1038/nature22786

Huotari, P. R. T., Nupponen, H., Laakso, L., and Kujala, U. M. (2010). Secular trends in aerobic fitness performance in 13-18-year-old adolescents from 1976 to 2001. Br. J. Sports Med. 44, 968-972. doi: 10.1136/bjsm.2008.055913

IEA (2016). Global Oil Supply Growth Plunging, with US Taking Biggest Hit for Now. Available online at: https://www.iea.org/newsroomandevents/pressreleases/ 2016/february/global-oil-supply-growth-plunging-with-us-taking-biggesthit-for-now.html. (Accessed October 18, 2016).

IPCC (2014). "Climate Change 2014: Impacts, Adaptation, and Vulnerability," in Summaries, Frequently Asked Questions, and Cross-Chapter Boxes. A Contribution of Working Group II to the Fifth Assessment Report of the Intergovernmental Panel on Climate Change, eds C. B. Field, V. R. Barros, D. J. Dokken, K. J. Mach, M. D. Mastrandrea, T. E. Bilir, M. Chatterjee, K. L. Ebi, Y. O. Estrada, R. C. Genova, B. Girma, E. S. Kissel, A. N. Levy, S. MacCracken, P. R. Mastrandrea, and L. L. White (Geneva: World Meteorological Organization), p. 190 (in Arabic, Chinese, English, French, Russian, and Spanish).

Justice, J. N., Cesari, M., Seals, D. R., Shively, C. A., and Carter, C. S. (2016). Comparative approaches to understanding the relation between aging and physical function. J. Gerontol. Ser. A 71, 1243-1253. doi: 10.1093/gerona/ glv035

Kannisto, V. (2000). Measuring the compression of mortality. Demogr. Res. 3:6. doi: 10.4054/DemRes.2000.3.6

Kennelly, A. E. (1906). An approximate law of fatigue in the speeds of racing animals. Proc. Am. Acad. Arts Sci. 42, 275-331. doi: 10.2307/20022230 
Kinugasa, R., and Usami, Y. (2016). How fast can a human run? Bipedal vs. Quadrupedal Running. Front. Bioeng. Biotechnol. 4:56. doi: 10.3389/fbioe.2016.00056

Kirkwood, T. B. L. (2017). Why and how are we living longer? Exp. Physiol. 102, 1067-1074. doi: 10.1113/EP086205

Kirkwood, T. B. L., and Austad, S. N. (2000). Why do we age? Nature 408, 233-238. doi: $10.1038 / 35041682$

Komlos, J. (2010). The recent decline in the height of African-American women. Econ. Hum. Biol. 8, 58-66. doi: 10.1016/j.ehb.2009.12.004

Komlos, J., and Baur, M. (2004). From the tallest to (one of) the fattest: the enigmatic fate of the American population in the 20th century. Econ. Hum. Biol. 2, 57-74. doi: 10.1016/j.ehb.2003.12.006

Komlos, J., and Lauderdale, B. E. (2007). The mysterious trend in American heights in the 20th century. Ann. Hum. Biol. 34, 206-215. doi: 10.1080/03014460601116803

Kuper, G. H., and Sterken, E. (2003). Endurance in speed skating: the development of world records. Eur. J. Oper. Res. 148, 293-301. doi: 10.1016/S0377-2217(02)00685-9

Kuper, G. H., and Sterken, E. (2007). "Modelling the development of world records in running," in Statistical Thinking in Sports, eds J. Albert and R. H. Koning (Boca Raton, FL: Chapman and Hall/CRC), 7-31.

Kurzweil, R., and Grossman, T. (2010). "Bridges to life," in The Future of Aging, eds G. M Fahy, D. M. D West, L. S. Coles, and S. B. Harris (New York, NY: Springer), 3-22.

Kusy, K., and Zielinski, J. (2014). Aerobic capacity in speed-power athletes aged 20-90 years vs endurance runners and untrained participants. Scand. J. Med. Sci. Sports 24, 68-79. doi: 10.1111/j.1600-0838.2012.01496.x

Laaidi, M., Laaidi, K., and Besancenot, J.-P. (2006). Temperature-related mortality in France, a comparison between regions with different climates from the perspective of global warming. Int. J. Biometeorol. 51, 145-153. doi: 10.1007/s00484-006-0045-8

Lanfear, R., Kokko, H., and Eyre-Walker, A. (2014). Population size and the rate of evolution. Trends Ecol. Evol. 29, 33-41. doi: 10.1016/j.tree.2013.09.009

Lang, J. J., Tremblay, M. S., Léger, L., Olds, T., and Tomkinson, G. R. (2016). International variability in $20 \mathrm{~m}$ shuttle run performance in children and youth: who are the fittest from a 50-country comparison? A systematic literature review with pooling of aggregate results. Br. J. Sports Med. doi: 10.1136/bjsports-2016-096224. [Epub ahead of print].

Larnkjær, A., Attrup Schrøder, S., Maria Schmidt, I., Hørby Jørgensen, M., and Fleischer Michaelsen, K. (2006). Secular change in adult stature has come to a halt in northern Europe and Italy. Acta Pcediatrica 95, 754-755. doi: 10.1080/08035250500527323

Larry, K. W., Jack, W., and David, C. (2012). Physiology of Sport and Exercise, 6th Edn. Champaign, IL: Human Kinetics.

Le Bourg, E. (2012). Forecasting continuously increasing life expectancy: what implications? Ageing Res. Rev. 11, 325-328. doi: 10.1016/j.arr.2012.01.002

Le Bourg, É. A. (2001). Mini-review of the evolutionary theories of aging: is it the time to accept them? Demogr. Res. 4, 1-28. doi: 10.4054/DemRes.2001.4.1

Le Bourg, E., and Vijg, J. (2017). The future of human longevity: time for a reality check. Gerontology 63, 527-528. doi: 10.1159/000478891

Lenart, A., and Vaupel, J. W. (2017). Questionable evidence for a limit to human lifespan. Nature 546, E13-E14. doi: 10.1038/nature22790

Li, J. S. H., Ng, A. C. Y., and Chan, W. S. (2011). Modeling old-age mortality risk for the populations of Australia and New Zealand: an extreme value approach. Math. Comput. Simul. 81, 1325-1333. doi: 10.1016/j.matcom.2010.04.025

Lieberman, D. E., and Bramble, D. M. (2007). The evolution of marathon running. Sports Med. 37, 288-290. doi: 10.2165/00007256-200737040-00004

Lippi, G., Banfi, G., Favaloro, E. J., Rittweger, J., and Maffulli, N. (2008). Updates on improvement of human athletic performance: focus on world records in athletics. Br. Med. Bull. 87, 7-15. doi: 10.1093/bmb/ldn029

Liu, Y., Paul, S., and Fu, F. H. (2012). Accomplishments and compromises in prediction research for world records and best performances in track and field and swimming. Meas. Phys. Educ. Exerc. Sci. 16, 167-182. doi: 10.1080/1091367X.2012.700252

Marck, A., Berthelot, G., Foulonneau, V., Marc, A., Antero-Jacquemin, J., Noirez, P., et al. (2016). Age-related changes in locomotor performance reveal a similar pattern for Caenorhabditis elegans, Mus domesticus, Canis familiaris, Equus caballus, and Homo sapiens. J. Gerontol. A Biol. Sci. Med. Sci. 72, 455-463. doi: 10.1093/gerona/glw136

Marijon, E., Tafflet, M., Antero-Jacquemin, J., El Helou, N., Berthelot, G., Celermajer, D. S., et al. (2013). Mortality of French participants in the Tour de France (1947-2012). Eur. Heart J. 4, 3145-3150. doi: 10.1093/eurheartj/eht347

Mason, J. W., Ramseth, D. J., Chanter, D. O., Moon, T. E., Goodman, D. B., Mendzelevski, B., et al. (2007). Electrocardiographic reference ranges derived from 79,743 ambulatory subjects. J. Electrocardiol. 40, 228.e8-234.e8. doi: 10.1016/j.jelectrocard.2006.09.003

Maxwell, S. L., Fuller, R. A., Brooks, T. M., and Watson, J. E. M. (2016). Biodiversity: the ravages of guns, nets and bulldozers. Nature 536, 143-145. doi: $10.1038 / 536143 a$

McMichael, A. (2014). Population health in the Anthropocene: Gains, losses and emerging trends. Anthr. Rev. 1, 44-56. doi: 10.1177/2053019613514035

Merilä, J., and Hendry, A. P. (2014). Climate change, adaptation, and phenotypic plasticity: the problem and the evidence. Evol. Appl. 7, 1-14. doi: 10.1111 /eva.12137

Mitchell, W. K., Williams, J., Atherton, P., Larvin, M., Lund, J., Narici, M., et al. (2012). Sarcopenia, dynapenia, and the impact of advancing age on human skeletal muscle size and strength; a quantitative review. Front. Physiol. 3:260. doi: 10.3389/fphys.2012.00260

Moore, D. H. (1975). A study of age group track and field records to relate age and running speed. Publ. Online 253, 264-265. doi: 10.1038/253264a0

NCD-RisC (2016). A century of trends in adult human height. eLife 5:e13410. doi: $10.7554 /$ eLife. 13410.002

Nes, B. M., Janszky, I., Wisløff, U., Støylen, A., and Karlsen, T. (2013). Agepredicted maximal heart rate in healthy subjects: the HUNT fitness study. Scand. J. Med. Sci. Sports 23, 697-704. doi: 10.1111/j.1600-0838.2012.01445.x

Neubauer, S., and Hublin, J.-J. (2012). The evolution of human brain development. Evol. Biol. 39, 568-586. doi: 10.1007/s11692-011-9156-1

Nevill, A. M., and Whyte, G. (2005). Are there limits to running world records? Med. Sci. Sports Exerc. 37, 1785-1788. doi: 10.1249/01.mss.0000181676.62054.79

Nevill, A. M., Whyte, G. P., Holder, R. L., and Peyrebrune, M. (2007). Are there limits to swimming world records? Int. J. Sports Med. 28, 1012-1017. doi: 10.1055/s-2007-965088

Nielsen, R., Akey, J. M., Jakobsson, M., Pritchard, J. K., Tishkoff, S., Willerslev, E., et al. (2017). Tracing the peopling of the world through genomics. Nature 541, 302-310. doi: 10.1038/nature21347

Noakes, T., and Spedding, M. (2012). Olympics: run for your life. Nature 487, 295-296. doi: 10.1038/487295a

Norton, K., and Olds, T. (2001). Morphological evolution of athletes over the 20th century. Sports Med. 31, 763-783. doi: 10.2165/00007256-200131110-00001

Oeppen, J., and Vaupel, J. W. (2002). Broken limits to life expectancy. Science 296, 1029-1031. doi: 10.1126/science. 1069675

Olshansky, S. J., and Carnes, B. A. (2009). "The Future of human longevity," in International Handbook of Population Aging, ed P. Uhlenberg (New York, NY: Springer), 731-745.

Olshansky, S. J., and Carnes, B. A. (2013). Zeno's paradox of immortality. Gerontology 59, 85-92. doi: 10.1159/000341225

Olshansky, S. J., Carnes, B. A., and Cassel, C. (1990). In search of Methuselah: estimating the upper limits to human longevity. Science 250, 634-640. doi: $10.1126 /$ science.2237414

Olshansky, S. J., Carnes, B. A., and Désesquelles, A. (2001). Prospects for human longevity. Science 291, 1491-1492. doi: 10.1126/science.291.5508.1491

Omran, A. R. (2005). The epidemiologic transition: a theory of the epidemiology of population change. Milbank Q. 83, 731-757. doi: 10.1111/j.1468-0009.2005.00398.x

Poole, D. C., and Erickson, H. H. (2011). Highly athletic terrestrial mammals: horses and dogs. Compr. Physiol. 1, 1-37. doi: 10.1002/cphy.c091001

Rashad, I. (2008). Height, health, and income in the US, 1984-2005. Econ. Hum. Biol. 6, 108-126. doi: 10.1016/j.ehb.2007.10.002

Rasmussen, F., Johansson, M., and Hansen, H. (1999). Trends in overweight and obesity among 18-year-old males in Sweden between 1971 and 1995. Acta Podiatrica 88, 431-437. doi: 10.1111/j.1651-2227.1999.tb01136.x

Reinboud, W. (2004). Linear models can't keep up with sport gender gap. Nature 432, 147; author reply 147. doi: 10.1038/432147a 
Roach, N. T., Venkadesan, M., Rainbow, M. J., and Lieberman, D. E. (2013). Elastic energy storage in the shoulder and the evolution of high-speed throwing in Homo. Nature 498, 483-486. doi: 10.1038/nature12267

Robine, J. M., and Vaupel, J. W. (2002). Emergence of supercentenarians in low-mortality countries. North Am. Actuar. J. 6, 54-63. doi: 10.1080/10920277.2002.10596057

Rockström, J., Steffen, W., Noone, K., Persson, A., Chapin, F. S., Lambin, E. F., et al. (2009). A safe operating space for humanity. Nature 461, 472-475. doi: $10.1038 / 461472 \mathrm{a}$

Rootzén, H., and Zholud, D. (2017). Human life is unlimited - but short. ArXiv170802184 Stat.

Rozing, M. P., Kirkwood, T. B. L., and Westendorp, R. G. J. (2017). Is there evidence for a limit to human lifespan? Nature 546, E11-E12. doi: $10.1038 /$ nature 22788

Ruff, C. B. (1991). Climate and body shape in hominid evolution. J. Hum. Evol. 21, 81-105. doi: 10.1016/0047-2484(91)90001-C

Runhaar, J., Collard, D. C., Singh, A. S., Kemper, H. C., van Mechelen, W., Chinapaw, M., et al. (2010). Motor fitness in Dutch youth: differences over a 26-year period (1980-2006). J. Sci. Med. Sport 13, 323-328. doi: 10.1016/j.jsams.2009.04.006

Santtila, M., Kyröläinen, H., Vasankari, T., Tiainen, S., Palvalin, K., Häkkinen, A., et al. (2006). Physical fitness profiles in young Finnish men during the years 1975-2004. Med. Sci. Sports Exerc. 38:1990. doi: 10.1249/01.mss.0000232023.28984.78

Schoenberg, J. B., Beck, G. J., and Bouhuys, A. (1978). Growth and decay of pulmonary function in healthy blacks and whites. Respir. Physiol. 33, 367-393. doi: 10.1016/0034-5687(78)90063-4

Schönbeck, Y., Talma, H., van Dommelen, P., Bakker, B., Buitendijk, S. E., HiraSing, R. A., et al. (2013). The world's tallest nation has stopped growing taller: the height of Dutch children from 1955 to 2009. Pediatr. Res. 73, 371-377. doi: 10.1038/pr.2012.189

Sedeaud, A., Marc, A., Schipman, J., Schaal, K., Danial, M., Guillaume, M., et al. (2014). Secular trend: morphology and performance. J. Sports Sci. 32, 1146-1154. doi: 10.1080/02640414.2014.889841

Shiels, M. S, Chernyavskiy, P, Anderson, W. F, Best, A. F, Haozous, E. A, Hartge, P., et al. (2017). Trends in premature mortality in the USA by sex, race, and ethnicity from 1999 to 2014: an analysis of death certificate data. Lancet 389, 1043-1054. doi: 10.1016/S0140-6736(17)30187-3

Speakman, J. R. (2005). Body size, energy metabolism and lifespan. J. Exp. Biol. 208, 1717-1730. doi: 10.1242/jeb.01556

Spedding, M., and Spedding, C. (2008). Drugs in sport: a scientist-athlete's perspective: from ambition to neurochemistry. Br. J. Pharmacol. 154, 496-501. doi: 10.1038/bjp.2008.163

Steffen, W., Crutzen, P. J., and McNeill, J. R. (2007). The anthropocene: are humans now overwhelming the great forces of nature. AMBIO J. Hum. Environ. 36, 614-621. doi: 10.1579/0044-7447(2007)36[614:TAAHNO]2.0.CO;2

Steffen, W., Persson, A., Deutsch, L., Zalasiewicz, J., Williams, M., Richardson, K., et al. (2011). The anthropocene: from global change to planetary stewardship. AMBIO 40, 739-761. doi: 10.1007/s13280-011-0185-x

Steffen, W., Richardson, K., Rockström, J., Cornell, S. E., Fetzer, I., Bennett, E. M., et al. (2015). Planetary boundaries: guiding human development on a changing planet. Science 347:1259855. doi: 10.1126/science.1259855

Stulp, G., and Barrett, L. (2016). Evolutionary perspectives on human height variation. Biol. Rev. 91, 206-234. doi: 10.1111/brv.12165

Tanaka, H., Monahan, K. D., and Seals, D. R. (2001). Age-predicted maximal heart rate revisited. J. Am. Coll. Cardiol. 37, 153-156. doi: 10.1016/S0735-1097(00)01054-8

Tatem, A. J., Guerra, C. A., Atkinson, P. M., and Hay, S. I. (2004). Momentous sprint at the 2156 Olympics? Nature 431:525. doi: 10.1038/431525a

Thatcher, A. R. (1999). The long-term pattern of adult mortality and the highest attained age. J. R. Stat. Soc. Ser. A Stat. Soc. 162, 5-43. doi: 10.1111/1467-985X.00119
Thibault, V., Guillaume, M., Berthelot, G., El Helou, N., Schaal, K., Quinquis, L., et al. (2010). Women and men in sport performance: the gender gap has not evolved since 1983. J. Sports Sci. Med. 9, 214-223.

Tomkinson, G. R. (2007). Global changes in anaerobic fitness test performance of children and adolescents (1958-2003). Scand. J. Med. Sci. Sports 17, 497-507. doi: 10.1111/j.1600-0838.2006.00569.x

Tomkinson, G. R., and Olds, T. S. (2007). Secular changes in pediatric aerobic fitness test performance: the global picture. Med. Sport Sci. 50, 46-66. doi: 10.1159/000101075

Tomkinson, G. R., Macfarlane, D., Noi, S., Kim, D. Y., Wang, Z., Hong, R., et al. (2012). Temporal changes in long-distance running performance of asian children between 1964 and 2009. Sports Med. 42, 267-279. doi: 10.2165/11599160-000000000-00000

United Nations (2016). The Sustainable Development Goals Report. United Nations.

Vaupel, J. W. (1997). "The average french baby may live 95 or 100 years," in Longevity: To the Limits and Beyond, eds J. M. Robine, J. W. Vaupel, B. Jeune, and M. Allard (Berlin; Heidelberg: Springer), 11-27.

Vaupel, J. W. (2010). Biodemography of human ageing. Nature 464, 536-542. doi: $10.1038 /$ nature 08984

Vijg, J., and Le Bourg, E. (2017). Aging and the inevitable limit to human life span. Gerontology 63, 432-434. doi: 10.1159/000477210

Watts, K. A., Dupuis, D. J., and Jones, B. L. (2006). An extreme value analysis of advanced age mortality data. North Am. Actuar. J. 10, 162-178. doi: 10.1080/10920277.2006.10597419

Weon, B. M., and Je, J. H. (2009). Theoretical estimation of maximum human lifespan. Biogerontology 10, 65-71. doi: 10.1007/s10522-008-9156-4

West, G. B., and Brown, J. H. (2005). The origin of allometric scaling laws in biology from genomes to ecosystems: towards a quantitative unifying theory of biological structure and organization. J. Exp. Biol. 208, 1575-1592 doi: 10.1242/jeb.01589

Weyand, P. G., Sandell, R. F., Prime, D. N. L., and Bundle, M. W. (2010). The biological limits to running speed are imposed from the ground up. J. Appl. Physiol. 108, 950-961. doi: 10.1152/japplphysiol.00947.2009

Whipp, B. J., and Ward, S. A. (1992). Will women soon outrun men? Nature 355, 25. doi: $10.1038 / 355025 \mathrm{a} 0$

Williams, A. G., and Folland, J. P. (2008). Similarity of polygenic profiles limits the potential for elite human physical performance. J. Physiol. 586, 113-121. doi: 10.1113 /jphysiol.2007.141887

Wilmoth, J. R. (1998). The future of human longevity: a demographer's perspective. Science 280, 395-397. doi: 10.1126/science.280.5362.395

Wilmoth, J. R. (2000). Demography of longevity: past, present, and future trends. Exp. Gerontol. 35, 1111-1129. doi: 10.1016/S0531-5565(00)00194-7

Wiswell, R. A., Hawkins, S. A., Jaque, S. V., Hyslop, D., Constantino, N., Tarpenning, K., et al. (2001). Relationship between physiological loss, performance decrement, and age in master athletes. J. Gerontol. A Biol. Sci. Med. Sci. 56, M618-M626. doi: 10.1093/gerona/56.10.M618

Zaatari, S. E., Grine, F. E., Ungar, P. S., and Hublin, J. J. (2016). Neandertal versus modern human dietary responses to climatic fluctuations. PLoS ONE 11:e0153277. doi: 10.1371/journal.pone.0153277

Conflict of Interest Statement: The authors declare that the research was conducted in the absence of any commercial or financial relationships that could be construed as a potential conflict of interest.

Copyright (C) 2017 Marck, Antero, Berthelot, Saulière, Jancovici, Masson-Delmotte, Boeuf, Spedding, Le Bourg and Toussaint. This is an open-access article distributed under the terms of the Creative Commons Attribution License (CC BY). The use, distribution or reproduction in other forums is permitted, provided the original author(s) or licensor are credited and that the original publication in this journal is cited, in accordance with accepted academic practice. No use, distribution or reproduction is permitted which does not comply with these terms. 